\title{
VOLTERRA EQUATIONS WITH FRACTIONAL STOCHASTIC INTEGRALS
}

\author{
MAHMOUD M. EL-BORAI, KHAIRIA EL-SAID EL-NADI, \\ OSAMA L. MOSTAFA, AND HAMDY M. AHMED
}

Received 24 December 2003 and in revised form 6 May 2004

Some fractional stochastic systems of integral equations are studied. The fractional stochastic Skorohod integrals are also studied. The existence and uniquness of the considered stochastic fractional systems are established. An application of the fractional BlackScholes is considered.

\section{Introduction}

We assume that a probability space $(\Omega, \eta, P)$ is given, where $\Omega$ denotes the space $C\left(\mathbb{R}_{+}\right.$, $\mathbb{R}^{k}$ ) equipped with the topology of uniform convergence on compact sets, $\eta$ the Borel $\sigma$-field of $\Omega$, and $P$ a probability measure on $\Omega$.

Let $\left\{W_{t}(\omega)=\omega(t), t \geq 0\right\}$ be a Wiener process. For any $t \geq 0$, we define $\eta_{t}=\sigma\{\omega(s)$; $s<t\} \vee Z$, where $Z$ denotes the class of the elements in $\eta_{t}$ which have zero $P$-measure.

Pardoux and Protter discussed the existence and uniqueness of the solution of the stochastic integral equation of the form

$$
X_{t}=X_{0}+\int_{0}^{t} F\left(t, s, X_{s}\right) d s+\sum_{i=1}^{k} \int_{0}^{t} G_{i}\left(H_{t} ; t, s, X_{s}\right) d W_{s}^{i},
$$

whose solution $\left\{X_{t}\right\}$ should be $\mathbb{R}^{d}$-valued and $\eta_{t}$ adapted process; $\left\{H_{t}\right\}$ is an $\mathbb{R}^{p}$-valued (see [16]). It is supposed that $F$ maps $\{t, s ; 0 \leq s<t\} \times \mathbb{R}^{d}$ into $\mathbb{R}^{d}$ and $G_{i}(i=1,2, \ldots, k)$ maps $\mathbb{R}^{p} \times\{t, s ; 0 \leq s<t\} \times \mathbb{R}^{d}$ into $\mathbb{R}^{d}$.

In the present work, we study the existence, uniqueness, and continuity of the solution of the fractional stochastic integral equation of the form

$$
X_{t}=X_{0}+I_{t}^{\beta} F\left(t, s, X_{s}\right)+\sum_{i=1}^{k} W_{t}^{\beta} G_{i}\left(H_{t} ; t, s, X_{s}\right),
$$

where $0<\beta<1, I_{t}^{\beta} F\left(t, s, X_{s}\right)$, the fractional integral of $F\left(t, s, X_{s}\right)$, is defined by (see [22])

$$
I_{t}^{\beta} F\left(t, s, X_{s}\right)=\frac{1}{\Gamma(\beta)} \int_{0}^{t} \frac{F\left(t, s, X_{s}\right)}{(t-s)^{1-\beta}} d s .
$$


The fractional Wiener process $W_{t}^{\beta} G_{i}\left(H_{t} ; t, s, X_{s}\right)$ of $G_{i}\left(H_{t} ; t, s, X_{s}\right)$ is defined by (see [7])

$$
W_{t}^{\beta} G_{i}\left(H_{t} ; t, s, X_{s}\right)=\frac{1}{\Gamma((\beta+1) / 2)} \int_{0}^{t} \frac{G_{i}\left(H_{t} ; t, s, X_{s}\right)}{(t-s)^{(1-\beta) / 2}} d W_{s}^{i} .
$$

Stochastic Volterra equations have been studied in several papers (see $[2,3,4,6,16,17$, 18]). In this work, we will use the Skorohod integral (see $[9,10,11,12,13,14,15,19,20]$ ) to interpret (1.2) as in [16] since the integrands in the stochastic integrals are not adapted; therefore we cannot use, as usual, the Ito integral to interpret the equation. In Section 2, we state some results concerning the Skorohod integral, which will be used later together with the precise interpretation of (1.2). In Sections 3 and 4, we prove the existence and uniqueness of a solution to (1.2) in two steps. In Section 5, we establish, under additional assumptions, the existence of almost surely (a.s.) continuous modification of the solution process. In Section 6, we show the continuity of the solution of (1.2).

Equation (1.2) has many important financial applications. These systems arise if we consider the fractional analog of a portfolio (see [1]). The fractional Black-Scholes market consists of a bank account or a bond and a stock. The price process $A_{t}$ of the bond at time $t$ is given by

$$
A_{t}=\exp \left\{\int_{0}^{t} r(s) d s\right\}
$$

where $r(s) \geq 0, s \in[0, t]$, is the interest rate. A portfolio is a pair $\left(u_{t}, v_{t}\right)$ of random variables for fixed $t \in[0, T]$. The price $X_{t}$ of the stock could be governed by a fractional Volterra equation of the form

$$
X_{t}=X_{0}+\frac{1}{\Gamma(\beta)} \int_{0}^{t} \frac{\mu(s) X_{s}}{(t-s)^{1-\beta}} d s+\frac{1}{\Gamma((\beta+1) / 2)} \int_{0}^{t} \frac{\sigma(s) X_{s}}{(t-s)^{(1-\beta) / 2}} d W_{s}
$$

Here the drift $\mu \geq 0$ and volatility $\sigma>0$ are continuous functions on $[0, T]$. The numbers $u_{t}$ and $v_{t}$ are the bond and stock units, respectively (held by an investor). Hence, the corresponding value process is

$$
V_{t}=u_{t} A_{t}+v_{t} X_{t}
$$

The process $V_{t}$ could be governed by the equation

$$
\begin{aligned}
V_{t}= & V_{0}+\frac{1}{\Gamma(\beta)} \int_{0}^{t} \frac{r(s) A_{s} u_{s}}{(t-s)^{1-\beta}} d s+\frac{1}{\Gamma(\beta)} \int_{0}^{t} \frac{\mu(s) X_{s} v_{s}}{(t-s)^{1-\beta}} d s \\
& +\frac{1}{\Gamma((1-\beta) / 2)} \int_{0}^{t} \frac{\sigma(s) v_{s} X_{s}}{(t-s)^{(1-\beta) / 2}} d W_{s} .
\end{aligned}
$$




\section{The Skorohod integral}

We will now define the Skorohod integral. Most of this section is a review of some basic notations and a few results from $[4,16]$.

Let, again, $\Omega=C\left(\mathbb{R}_{+}, \mathbb{R}^{k}\right)$, let $\eta$ be its Borel field, and let $P$ denote Wiener measure on $(\Omega, \eta)$

$$
W_{t}(\omega)=\omega(t)
$$

Let $\eta_{t}^{0}=\sigma\left\{W_{s} ; 0 \leq s<t\right\}$ and $\eta_{t}=\eta_{t}^{0} \vee Z$, where $Z$ denotes the class of sets which have zero $P$-measure of $\eta$.

For $h \in L^{2}\left(\mathbb{R}_{+} ; \mathbb{R}^{k}\right)$, we denote the Wiener integral by

$$
W(h)=\int_{0}^{T}\left(h(t), d W_{t}\right)
$$

Let $A$ denote the dense subset of $L^{2}(\Omega, \eta, P)$ consisting of those classes of random variables of the form

$$
F=f\left(W\left(h_{1}\right), \ldots, W\left(h_{n}\right)\right)
$$

where $n \in \mathbb{N}$ ( $\mathbb{N}$ denotes the set of nonnegative integers), $f \in C_{b}^{\infty}\left(\mathbb{R}^{n}\right), h_{1}, \ldots, h_{n} \in$ $L^{2}\left(\mathbb{R}_{+} ; \mathbb{R}^{k}\right) ; C_{b}^{\infty}$ is the set of infinitely differentiable functions on $[0, b]$ whose derivatives of any order are null at $b$. If $F$ has the form (2.3), we define its derivative in the direction $i$ as the process $\left\{D_{t}^{i} F ; t \geq 0\right\}$ defined by

$$
D_{t}^{i} F=\sum_{k=1}^{n} \frac{\partial f}{\partial x_{k}}\left(W\left(h_{1}\right), \ldots, W\left(h_{n}\right)\right) h_{k}^{i}(t) .
$$

$D F$ will stand for the $k$-dimensional process $\left\{D_{t} F=\left(D_{t}^{1} F, \ldots, D_{t}^{k} F\right) ; t \geq 0\right\}$.

Proposition 2.1. The differential operators $D^{i}, i=1, \ldots, k$, are unbounded closable operators from $L^{2}(\Omega)$ into $L^{2}\left(\Omega \times \mathbb{R}_{+}\right)$.

Let $D_{i}^{1,2}$ be the closure of $A$ with respect to the norm

$$
\|F\|_{i, 1,2}=\|F\|_{2}+\|\cdot\| D^{i} F\left\|_{L^{2}\left(\mathbb{R}_{+}\right)}\right\|_{2}
$$

where $\|F\|_{2}=\left(E\left(F^{2}\right)\right)^{1 / 2}, E(X)$ is the mathematical expectation of $X$, and

$$
\|g\|_{L^{2}\left(\mathbb{R}_{+}\right)}^{2}=\int_{0}^{\infty} g^{2}(t) d t
$$

Similarly, the domain $D^{1,2}=\bigcap_{i=1}^{k} D_{i}^{1,2}$ is the closure of $A$ with respect to the norm (see [10])

$$
\|F\|_{1,2}=\|F\|_{2}+\sum_{i=1}^{k}\|\cdot\| D^{i} F\left\|_{L^{2}\left(\mathbb{R}_{+}\right)}\right\|_{2} .
$$


We identify $D^{i}$ and $D$ with their closed extensions $\left(D^{1,2}\right.$ is the domain of $D: L^{2}(\Omega) \rightarrow$ $\left.L^{2}\left(\Omega \times \mathbb{R}_{+} ; \mathbb{R}^{k}\right)\right)$.

We denote by $D_{i, \text { loc }}^{1,2}$ the set of measurable $F$ 's which are such that there exists a sequence $\left\{\left(\Omega_{n}, F_{n}\right) ; n \in \mathbb{N}\right\} \subset \eta \times D_{i}^{1,2}$ with the two properties

(i) $\Omega_{n} \uparrow \Omega$ a.s., $n \rightarrow \infty$,

(ii) $F_{n}\left|\Omega_{n}=F\right|_{\Omega_{n}}, n \in \mathbb{N}$.

For $\mathrm{F} \in D_{i, \text { loc }}^{1,2}$, we define without ambiguity $D_{t}^{i} F=D_{t}^{i} F_{n}$ on $\Omega_{n} \times \mathbb{R}_{+}$for all $n \in \mathbb{N} ; D_{\text {loc }}^{1,2}$ is defined similarly.

For $i=1, \ldots, k$, we define $\delta_{i}$, the Skorohod integral with respect to $W_{t}^{i}$, as the adjoint of $D^{i}$, that is, Dom $\delta_{i}$ (the set of adapted processes for the Skorohod integral) is the set of $u \in L^{2}\left(\Omega \times \mathbb{R}_{+}\right)$which are such that there exists a constant $c$ with

$$
\left|E \int_{0}^{\infty} D_{t}^{i} F u_{t} d t\right| \leq c\|F\|_{2}, \quad \forall F \in A
$$

If $u \in \operatorname{Dom} \delta_{i}, \delta_{i}(u)$ is defined as the unique element of $L^{2}(\Omega)$ which satisfies

$$
E\left(\delta_{i}(u) F\right)=E \int_{0}^{\infty} D_{t}^{i} F u_{t} d t, \quad \forall F \in A
$$

Let $L_{i}^{1,2}=L^{2}\left(\mathbb{R}_{+} ; D_{i}^{1,2}\right)$. We have that $L_{i}^{1,2} \subset \operatorname{Dom} \delta_{i}$, and, for $u \in L_{i}^{1,2}$,

$$
E\left[\delta_{i}(u)^{2}\right]=E \int_{0}^{\infty} u_{t}^{2} d t+E \int_{0}^{\infty} \int_{0}^{\infty} D_{s}^{i} u_{t} D_{t}^{i} u_{s} d s d t
$$

Note that if $u \in L_{\text {loc }}^{2}\left(\mathbb{R}_{+} ; D_{i}^{1,2}\right)$, then $u 1_{[0, T]} \in L_{i}^{1,2}$ for any $T>0$ and we can write

$$
\int_{0}^{T} u_{t} d W_{t}^{i}=\delta_{i}\left(u 1_{[0, T]}\right)
$$

The Skorohod integral is a local operation on $L_{\text {loc }}^{2}\left(\mathbb{R}_{+} ; D_{i}^{1,2}\right)$ in the sense that if $u, v \in$ $L_{\text {loc }}^{2}\left(\mathbb{R}_{+} ; D_{i}^{1,2}\right)$, then $\int_{0}^{t} u_{s} d W_{s}^{i}=\int_{0}^{t} v_{s} d W_{s}^{i}$ a.s. on $\left\{\omega ; u_{s}(\omega)=v_{s}(\omega)\right.$, for almost all $\left.s<t\right\}$.

Let $L_{i, \text { loc }}^{1,2}$ denote the set of measurable processes $u$ which are such that, for any $T>0$, there exists a sequence

$$
\left\{\left(\Omega_{n}^{T}, u_{n}^{T}\right) ; n \in \mathbb{N}\right\} \subset \eta \times L_{i}^{1,2}
$$

such that

(i) $\Omega_{n}^{T} \uparrow \Omega$ a.s., as $n \rightarrow \infty$,

(ii) $u=u_{n}^{T} d P \times d t$ a.e. on $\Omega_{n}^{T} \times[0, T], n \in \mathbb{N}$. 
For $u \in L_{i, \text { loc }}^{1,2}$, we can define its Skorohod integral with respect to $W_{t}^{i}$ by

$$
\int_{0}^{t} u_{s} d W_{s}^{i}=\int_{0}^{t} u_{n, s}^{T} d W_{s}^{i} \quad \text { on } \Omega_{n}^{T} \times[0, T]
$$

Finally, $L^{1,2}=\bigcap_{i=1}^{k} L_{i}^{1,2}$, and $L_{\text {loc }}^{1,2}$ is defined similarly as $L_{i, \text { loc }}^{1,2}$.

We now introduce the particular class of integrands which we will use below.

Let $u: \mathbb{R}_{+} \times \Omega \times \mathbb{R}^{P} \rightarrow \mathbb{R}$ satisfy the following.

(i) For all $x \in \mathbb{R}^{p},(t, \omega) \rightarrow(t, \omega, x)$ is $\eta_{t}$ progressively measurable.

(ii) For all $(t, \omega) \in \mathbb{R}_{+} \times \Omega, u(t, \omega, \cdot) \in C^{1}\left(\mathbb{R}^{p}\right)$.

(iii) For some increasing function $\phi: \mathbb{R}_{+} \rightarrow \mathbb{R}_{+},|u(t, \omega, x)|+\left|u^{\prime}(t, \omega, x)\right| \leq \phi(|x|)$ for all $(t, \omega, x) \in \mathbb{R}_{+} \times \Omega \times \mathbb{R}^{p}$, where $u^{\prime}(t, x)$ stands for the gradient $(\partial u / \partial x)(t, x)$.

Let $\theta$ be a $p$-dimensional random vector such that

(iv) $\theta^{j} \in D_{i}^{1,2} \cap L^{\infty}(\Omega), j=1, \ldots, p$.

We fix $T>0$ and consider

$$
I^{i}(x)=\int_{0}^{T} \frac{u(t, x)}{(T-t)^{(1-\beta) / 2}} d W_{t}^{i}, \quad 0<\beta<1 .
$$

Define, moreover, $v_{t}=u(t, \theta)$.

Under conditions (i), (ii), (iii), and (iv), the following proposition holds.

It is proved in [10] that

$$
\int_{0}^{T} u(t, \theta) d W_{t}^{i}=\left.\int_{0}^{T} u(t, x) d W_{t}^{i}\right|_{x=\theta}-\int_{0}^{T} u^{\prime}(t, \theta) D_{t}^{i} \theta d t
$$

The same relation is proved in [16] under slightly different conditions. Equation (2.15) is used to define the Skorohod integral $\int_{0}^{T} u(t, \theta) d W_{t}^{i}$.

Proposition 2.2. The random field $\left\{I^{i}(x) ; x \in \mathbb{R}^{p}\right\}$ defined above possesses an a.s. continuous modification so that the random variable $I^{i}(\theta)$ can be defined, $v \in \operatorname{Dom} \delta_{i}($ see $[10,16])$.

Condition (iv) can be replaced by (iv'): $\theta^{j} \in D_{i, \text { loc }}^{1,2}, j=1, \ldots, p$.

Under conditions (i), (ii), (iii), and (iv'), $v \in\left(\operatorname{Dom} \delta_{i}\right)_{\text {loc }}$ in the sense that there exists a sequence $\left\{\left(\Omega_{n}, v_{n}\right) ; n \in \mathbb{N}\right\} \subset \eta \times \operatorname{Dom} \delta_{i}$ such that $\Omega_{n} \uparrow \Omega$ a.s. and $v_{n}\left|\Omega_{n}=v\right| \Omega_{n}$.

Indeed, let $\left\{\left(\Omega_{n}^{\prime}, \theta_{n}\right)\right\}$ be a localizing sequence for $\theta$ in $\left(D_{i}^{1,2}\right)^{p}$, and let $\left\{\psi_{n} ; n \in \mathbb{N}\right\} \subset$ $C_{c}^{\infty}\left(\mathbb{R}^{p} ; \mathbb{R}^{p}\right)$ satisfy $\psi_{n}(x)=x$ whenever $|x| \leq n$.

Define $v_{n}(t)=u\left(t, \psi_{n}\left(\theta_{n}\right)\right), \Omega_{n}=\Omega_{n}^{\prime} \bigcap\{|\theta| \leq n\}$; then $\left\{\left(\Omega_{n}, v_{n}\right) ; n \in \mathbb{N}\right\}$ satisfies the above conditions.

It is then natural to define, for every $\epsilon>0$, the Skorohod integral $\int_{0}^{T-\epsilon}\left(v_{t} /(T-t)^{(1-\beta) / 2}\right)$ $d W_{t}^{i}$ by formula (2.15) and the latter coincides with $\int_{0}^{T-\epsilon}\left(v_{n}(t) /(T-t)^{(1-\beta) / 2}\right) d W_{t}^{i}$ on $\Omega_{n}$.

It is clear that $\lim _{\epsilon \rightarrow 0} \int_{0}^{T-\epsilon}\left(v_{t} /(T-t)^{(1-\beta) / 2}\right) d W_{t}^{i}$ exists in the mean by using the norm $\|\cdot\|_{2}$. 


\section{Statement of the problem: interpretation of (1.2)}

Our aim is to study the equation

$$
\begin{gathered}
X_{t}=X_{0}+a \int_{0}^{t} \frac{F\left(t, s, X_{s}\right)}{(t-s)^{1-\beta}} d s+b \sum_{i=1}^{k} \int_{0}^{t} \frac{G_{i}\left(H_{t} ; t, s, X_{s}\right)}{(t-s)^{(1-\beta) / 2}} d W_{s}^{i}, \\
0<\beta<1, \quad a=\frac{1}{\Gamma(\beta)}, \quad b=\frac{1}{\Gamma((\beta+1) / 2)} .
\end{gathered}
$$

We define $D=\left\{(t, s) \in \mathbb{R}_{+}^{2} ; 0 \leq s<t\right\}$.

The coefficients $F$ and $G$ are given as follows: $F: D \times \mathbb{R}^{d} \rightarrow \mathbb{R}^{d}$ is measurable and, for each $(s, x) \in \mathbb{R}_{+} \times \mathbb{R}^{d}, F(\cdot, s, x)$ is $\eta_{t}$ progressively measurable on $\Omega \times[s,+\infty)$.

For $i=1, \ldots, k, G_{i}: \mathbb{R}^{p} \times D \times \mathbb{R}^{d} \rightarrow \mathbb{R}^{d}$ is measurable, for each $(h, t, x), G_{i}(h ; t, \cdot, x)$ is $\eta_{s}$ progressively measurable on $\Omega \times[0, t]$, and for each $(\omega, t, s, x), G_{i}(\cdot ; t, s, x)$ is of class $C^{1}$. $\left\{H_{t}\right\}$ is a given progressively measurable $p$-dimensional process. It will follow from these hypotheses that we will be able to construct a progressively measurable solution $\left\{X_{t}\right\}$.

Therefore, for each $t$, the process $\left\{G_{i}\left(h ; t, s, X_{s}\right) ; s \in[0, T]\right\}$ is of the form $v_{s}=u(s, \theta)$ with $u(s, h)=G_{i}\left(h ; t, s, X_{s}\right)$ and $\theta=H_{t}$. We will impose below conditions on $G,\left\{H_{t}\right\}$, and the solution $\left\{X_{t}\right\}$ so as to satisfy requirements (i), (ii), (iii), and (iv') of Section 2.

In particular, we will consider only nonanticipating solutions. Therefore, the stochastic integrals in (3.1) will be interpreted according to (2.15), that is,

$$
\int_{0}^{t} \frac{G_{i}\left(H_{t} ; t, s, X_{s}\right)}{(t-s)^{(1-\beta) / 2}} d W_{s}^{i}=\left.\int_{0}^{t} \frac{G_{i}\left(h ; t, s, X_{s}\right)}{(t-s)^{(1-\beta) / 2}} d W_{s}^{i}\right|_{h=H_{t}}-\int_{0}^{t} \frac{G_{i}^{\prime}\left(H_{t} ; t, s, X_{s}\right)}{(t-s)^{(1-\beta) / 2}} D_{s}^{i} H_{t} d s .
$$

In other words, we can rewrite (3.1) as

$$
X_{t}=X_{0}+\int_{0}^{t} \tilde{F}\left(t, s, X_{s}\right) d s+\left.b \sum_{i=1}^{k} \int_{0}^{t} \frac{G_{i}\left(h ; t, s, X_{s}\right)}{(t-s)^{(1-\beta) / 2}} d W_{s}^{i}\right|_{h=H_{t}}
$$

where

$$
\tilde{F}(t, s, x)=a \frac{F(t, s, x)}{(t-s)^{1-\beta}}-b \sum_{i=1}^{k} \frac{G_{i}^{\prime}\left(H_{t} ; t, s, x\right) D_{s}^{i} H_{t}}{(t-s)^{(1-\beta) / 2}},
$$

and the stochastic integrals are now the usual Itô integrals.

We will show below that (3.3) makes sense for any progressively measurable process $X$ which satisfies $X \in \bigcap_{t>0} L^{q}(0, t)$ a.s., for some $q>p$. We will find such a solution to (3.3); it will then follow from (3.2) that it is a solution to (3.1). Similarly, uniqueness for (3.1) in the above class will follow from uniqueness for (3.3) in that class.

\section{Existence and uniqueness under strong hypotheses}

We formulate a set of further hypotheses (those stated in Section 3 are assumed to hold throughout the paper) under which we will establish a first result of the existence and uniqueness of a solution of (1.2). 
Let $B$ be an open bounded subset of $\mathbb{R}^{p}, K>0$, and $q>p$ such that

(H1) $X_{0} \in L^{q}\left(\Omega, \eta_{0}, P ; \mathbb{R}^{d}\right)$,

(H2) $P\left(H_{t} \in B, \forall t \geq 0\right)=1$,

(H3) $H \in\left(L^{1,2}\right)^{p},\left|D_{s} H_{t}\right| \leq K$ a.s., $0 \leq s<t$,

(H4) $|F(t, s, x)|+\sum_{i=1}^{k}\left|G_{i}(h ; t, s, x)\right|+\sum_{i=1}^{k}\left|G_{i}^{\prime}(h ; t, s, x)\right| \leq K(1+|x|)$, for any $0 \leq s<t$, $h \in B, x \in \mathbb{R}^{d}$

(H5) $|F(t, s, x)-F(t, s, y)|+\sum_{i=1}^{k}\left|G_{i}(h ; t, s, x)-G_{i}(h ; t, s, y)\right|+\sum_{i=1}^{k} \mid G_{i}^{\prime}(h ; t, s, x)$ $-G_{i}^{\prime}(h ; t, s, y)|\leq K| x-y \mid$, for any $0 \leq s<t, h \in B$, and $x, y \in \mathbb{R}^{d}$ a.s.

Note that $q$ will be a fixed real number such that $q>p$, and $L_{\mathrm{prog}}^{q}(\Omega \times(0, t))$ will stand for the space $L^{q}\left(\Omega \times(0, t), \xi_{t}, P \times \lambda\right)$, where $\xi_{t}$ denotes the $\sigma$-algebra of progressively measurable subsets of $\Omega \times(0, t)$ and $\lambda$ denotes the Lebesgue measure on $(0, t)$, and set $1-\beta=\alpha / q, 0<\alpha<1$.

Lemma 4.1. Let $X \in \bigcap_{t>0} L_{\text {prog }}^{q}(\Omega \times(0, t))$, where $q>p$, and suppose that $(H .4)$ is in force. Then for any $T \geq t>0$ and $i \in\{1, \ldots, k\}$, the random field

$$
\left\{\int_{0}^{t} \frac{G_{i}\left(h ; t, s, X_{s}\right)}{(t-s)^{\alpha / 2 q}} d W_{s}^{i} ; h \in B\right\}
$$

possesses an a.s. continuous modification.

Proof. Using Burkholder-Gundy and Hölder's inequalities together with (H.4), we obtain

$$
\begin{aligned}
& E\left(\left|\int_{0}^{t} \frac{G_{i}\left(h ; t, s, X_{s}\right)}{(t-s)^{\alpha / 2 q}} d W_{s}^{i}-\int_{0}^{t} \frac{G_{i}\left(k ; t, s, X_{s}\right)}{(t-s)^{\alpha / 2 q}} d W_{s}^{i}\right|^{q}\right) \\
& \quad \leq c_{1} E \int_{0}^{t} \frac{\left|G_{i}\left(h ; t, s, X_{s}\right)-G_{i}\left(k ; t, s, X_{s}\right)\right|^{q}}{(t-s)^{\alpha / 2}} d s \\
& \quad \leq c_{1} K^{q}|h-k|^{q} \int_{0}^{t} \frac{E\left(1+\left|X_{s}\right|^{q}\right)}{(t-s)^{\alpha / 2}} d s \\
& \quad \leq c_{2} K^{q}|h-k|^{q} \int_{0}^{t}(t-s)^{-\alpha / 2} d s \\
& \quad \leq c_{3}|h-k|^{q} t^{1-\alpha / 2},
\end{aligned}
$$

where $c_{1}, c_{2}$, and $c_{3}$ are positive constants. The result now follows from the multidimensional generalization of Kolmogrov's lemma (see $[1,8]$ ).

We can now assume that, for fixed $t$, the random field (4.1) is a.s. continuous in $h$, provided $X \in L_{\text {prog }}^{q}(\Omega \times(0, t))$.

From $X \in \bigcap_{t>0} L_{\text {prog }}^{q}(\Omega \times(0, t))$, define

$$
\begin{aligned}
I_{t}(X, h) & =b \sum_{i=1}^{k} \int_{0}^{t} \frac{G_{i}\left(h ; t, s, X_{s}\right)}{(t-s)^{\alpha / 2 q}} d W_{s}^{i}, \quad h \in \mathbb{R}^{p}, t>0, \\
J_{t}(X) & =\int_{0}^{t} \tilde{F}\left(t, s, X_{s}\right) d s+I_{t}\left(X, H_{t}\right) .
\end{aligned}
$$


LEMma 4.2. For any $t>0$, there is a constant $c>0$ such that

$$
E\left(\left|J_{t}(X)\right|^{q}\right) \leq c\left(\int_{0}^{t} E\left(1+\left|X_{s}\right|^{q}\right)\left\{\frac{1}{(t-s)^{\alpha}}+\frac{1}{(t-s)^{\alpha / 2}}\right\} d s\right), \quad 0<\alpha<1 .
$$

Proof.

$$
\begin{aligned}
\left|\int_{0}^{t} \tilde{F}\left(t, s, X_{s}\right) d s\right|^{q} & \leq c \int_{0}^{t} \frac{\left|F\left(t, s, X_{s}\right)\right|^{q}}{(t-s)^{\alpha}}+\sum_{i=1}^{k} \frac{\left|G_{i}^{\prime}\left(h ; t, s, X_{s}\right)\right|_{h=H_{t}}^{q}\left|D_{s}^{i} H_{t}\right|^{q}}{(t-s)^{\alpha / 2}} d s \\
& \leq \tilde{c} \int_{0}^{t}\left(1+\left|X_{s}\right|^{q}\right)\left\{\frac{1}{(t-s)^{\alpha}}+\frac{1}{(t-s)^{\alpha / 2}}\right\} d s,
\end{aligned}
$$

where we have used (H.2), (H.3), and (H.4).

$$
\left|I_{t}\left(X, H_{t}\right)\right| \leq \sup _{h \in B}\left|I_{t}(X, h)\right|
$$

(c, $\tilde{c}$ are positive constants).

It is easy to show, using, in particular, $(\mathrm{H} 4)$ and Lebesgue's dominated convergence theorem, that the mapping

$$
h \longrightarrow I_{t}(X, h)
$$

from $\mathbb{R}^{p}$ into $L^{q}(\Omega)$ is differentiable and that

$$
\frac{\partial I_{t}(X, h)}{\partial h_{j}}=b \int_{0}^{t} \frac{\partial}{\partial h_{j}} \sum_{i=1}^{k}\left(\frac{G_{i}\left(h ; t, s, X_{s}\right)}{(t-s)^{\alpha / 2 q}}\right) d W_{s}^{i} .
$$

Since $q>p$, we can infer from Sobolev's embedding theorem (see [13]) that

$$
E\left(\sup _{h \in B}\left|I_{t}(X, h)\right|^{q}\right) \leq c E \int_{B}\left(\left|I_{t}(X, h)\right|^{q}+\sum_{j=1}^{p}\left|\frac{\partial I_{t}}{\partial h_{j}}(X, h)\right|^{q}\right) d h .
$$

It then follows from the Burkholder-Gundy inequality that

$$
\begin{aligned}
E\left(\sup _{h \in B}\left|I_{t}(X, h)\right|^{q}\right) & \leq c E \int_{B} \int_{0}^{t} \sum_{i=1}^{k}\left(\frac{\left|G_{i}\left(h ; t, s, X_{s}\right)\right|^{q}}{(t-s)^{\alpha / 2}}+\sum_{j=1}^{p}\left|\frac{\partial}{\partial h_{j}} \frac{G_{i}\left(h ; t, s, X_{s}\right)}{(t-s)^{\alpha / 2 q}}\right|^{q}\right) d s d h \\
& \leq c\left(\int_{B} d h\right)\left(E \int_{0}^{t} \frac{\left(1+\left|X_{s}\right|^{q}\right)}{(t-s)^{\alpha / 2}} d s\right) \\
& \leq \tilde{c}\left\{\int_{0}^{t} \frac{E\left(1+\left|X_{s}\right|^{q}\right)}{(t-s)^{\alpha / 2}} d s\right\},
\end{aligned}
$$

where we have used (H4) and $B$ is bounded. From (4.5) and (4.10), the proof is complete.

A similar argument, using (H5) instead of (H4), yields the following lemma. 
Lemma 4.3. For any $0<t \leq T$, there exists a constant $c>0$ such that

$$
E\left(\left|J_{t}(X)-J_{t}(Y)\right|^{q}\right) \leq c\left\{\int_{0}^{t} E\left|X_{s}-Y_{s}\right|^{q}\left\{\frac{1}{(t-s)^{\alpha}}+\frac{1}{(t-s)^{\alpha / 2}}\right\} d s\right\} .
$$

We are now in a position to prove the main result of this section.

Theorem 4.4. Under conditions (H.1), (H.2), (H.3), (H.4), and (H.5), there exists a unique element $X \in \bigcap_{t>0} L_{\text {prog }}^{q}(\Omega \times(0, t))$, which solves (3.1). Moreover, if $\tau$ is a stopping time, uniqueness holds on the random interval $[0, \tau]$.

Proof. Equation (3.1) can be rewritten as

$$
X_{t}=X_{0}+J_{t}(X), \quad t \geq 0 .
$$

Uniqueness. Let $X, Y \in \bigcap_{t>0} L_{\text {prog }}^{q}(\Omega \times(0, t))$ and let $\tau$ be a stopping time such that

$$
X_{t}=X_{0}+J_{t}(X), \quad Y_{t}=X_{0}+J_{t}(Y), \quad 0 \leq t \leq \tau .
$$

From Lemma 4.3,

$$
\begin{aligned}
E\left(\left|X_{t}-Y_{t}\right|^{q}\right) & =E\left(\left|J_{t}(X)-J_{t}(Y)\right|^{q}\right) \\
& \leq c \int_{0}^{t} E\left|X_{s}-Y_{s}\right|^{q}\left\{\frac{1}{(t-s)^{\alpha}}+\frac{1}{(t-s)^{\alpha / 2}}\right\} d s .
\end{aligned}
$$

Set $\left(E\left|X_{t}-Y_{t}\right|^{q}\right)=\gamma_{t}$. It is easy to see that

$$
\gamma_{t} \leq 2 c \eta(t) \int_{0}^{t} \frac{\gamma_{s}}{(t-s)^{\alpha}} d s
$$

where $\eta(t)=\operatorname{Max}\left(1, t^{\alpha}\right)$ and $c$ is a positive constant. Thus, (see $\left.[5,21]\right)$

$$
\gamma_{t} \leq \frac{M^{2}(\Gamma(t-\alpha))^{2}}{\Gamma(2(1-\alpha))} \int_{0}^{t} \frac{\gamma_{s}}{(t-s)^{2 \alpha-1}} d s,
$$

and, by a classical argument, we get

$$
\gamma_{t} \leq \frac{M^{n}(\Gamma(t-\alpha))^{n}}{\Gamma(n(1-\alpha))} \int_{0}^{t} \frac{\gamma_{s}}{(t-s)^{n \alpha-(n-1)}} d s,
$$

where $M=2 c \eta(T)$. Thus for sufficiently large $n, n>1 /(1-\alpha)$, we get, from (4.17),

$$
\gamma_{t} \leq \frac{K^{n}}{\Gamma(n(1-\alpha))} \int_{0}^{t} \gamma_{s} d s
$$

where $K$ is a positive constant.

Taking the limit as $n \rightarrow \infty$, we find that (4.18) leads to $\gamma_{t}=0$.

To prove the existence, we define a sequence $\left\{X_{t}^{n}, 0 \leq t \leq T, n=0,1,2, \ldots\right\}$ as follows:

$$
X_{t}^{o}=X_{o}, \quad X_{t}^{n+1}=X_{o}+J_{t}\left(X^{n}\right), \quad T \leq t \leq 0 .
$$


462 Volterra equations with fractional stochastic integrals

Using Lemma 4.2, we can see that

$$
X^{n} \in \bigcap_{t>0} L_{\text {prog }}^{q}(\Omega \times[0, T]) .
$$

It then follows from Lemma 4.3 that

$$
g_{n+1}(t) \leq 2 c \eta(t) \int_{0}^{t} \frac{g_{n}(s)}{(t-s)^{\alpha}} d s
$$

where

$$
g_{n+1}(t)=E\left(\left|X_{t}^{n+1}-X_{t}^{n}\right|^{q}\right)
$$

By using a similar argument, we can write

$$
g_{n} \leq \frac{K^{n}}{\Gamma(n(1-\alpha))} \int_{0}^{t} g_{o}(s) d s .
$$

The last estimation implies that $X^{n}$ is a Cauchy sequence in $L_{\mathrm{prog}}^{q}(\Omega \times[0, T])$. Then there exists $X$ such that $X^{n} \rightarrow X$ in $\bigcap_{t>0} L_{\text {prog }}^{q}(\Omega \times[0, T])$, and again using Lemma 4.3, we can pass to the limit in (4.20), yielding that $X$ solves (4.12).

\section{An existence and uniqueness result under weaker assumptions}

We formulate a new set of weaker hypotheses.

$\left(\mathrm{H} 1^{\prime}\right) X_{0}$ is $\eta_{0}$ measurable.

$\left(\mathrm{H} 2^{\prime}\right) H \in\left(L_{\mathrm{loc}}^{1,2}\right) p,\left\{H_{t}\right\}$ is a progressively measurable process which can be localized in $\left(L^{1,2}\right)^{p}$ by a progressively measurable sequence.

We assume that there exists an increasing progressively measurable process $\left\{U_{t}, t \geq 0\right\}$ with values in $\mathbb{R}_{+}$such that

$$
\left(\mathrm{H}^{\prime}\right)\left|H_{t}\right|+\sum_{i=1}^{k}\left|D_{s}^{i} H_{t}\right| \leq U_{t} \text { a.s., } 0 \leq s<t \text {. }
$$

Finally, we suppose that for any $\mathbb{N}>0$, there exists an increasing progressively measurable process $\left\{V_{t}^{N} ; t \geq 0\right\}$ with values in $\mathbb{R}_{+}$such that

$$
\begin{aligned}
\left(\mathrm{H}^{\prime}\right) & |F(t, s, x)|+\sum_{i=1}^{k}\left|G_{i}(h ; t, s, x)\right|+\sum_{i=1}^{k}\left|G_{i}^{\prime}(h ; t, s, x)\right| \leq V_{t}^{N}(1+|x|), \text { for all }|h| \leq N, \\
& 0 \leq s<t \text {, and } x \in \mathbb{R}^{d} ; \\
\left(\mathrm{H}^{\prime}\right) & |F(t, s, x)-F(t, s, y)|+\sum_{i=1}^{k}\left|G_{i}(h ; t, s, x)-G_{i}(h ; t, s, y)\right|+\sum_{i=1}^{k} \mid G_{i}^{\prime}(h ; t, s, x) \\
& -G_{i}^{\prime}(h ; t, s, y)\left|\leq V_{t}^{N}\right| x-y \mid, \text { for all }|h| \leq N, 0 \leq s<t, x, y \in \mathbb{R}^{d} .
\end{aligned}
$$

Let, again, $q$ be a fixed real number, with $q>p$, and set $1-\beta=\alpha / q, 0<\alpha<1$. We have the following theorem.

TheOREM 5.1. Equation (3.1) has a unique solution in the class of progressively measurable processes which satisfy

$$
X \in \bigcap_{t>0} L^{q}(0, t) \quad \text { a.s. }
$$


Proof. (a) We first see how (3.1) makes sense if $X \in \bigcap_{t>0} L^{q}(0, t)$ a.s.

That is, we have to show that for fixed $t>0$,

$$
\left\{\int_{0}^{t} \frac{G_{i}\left(h ; t, s, X_{s}\right)}{(t-s)^{\alpha / 2 q}} d W_{s}^{i} ; h \in \mathbb{R}^{P}\right\}
$$

is a well-defined random field which possesses an a.s. continuous version.

For that sake, we define

$$
\tau_{n}=\inf \left\{t ; \int_{0}^{t} \frac{\left|X_{s}\right|^{q}}{(t-s)^{\alpha}} d s \geq n \text { or } V_{t}^{N} \geq n\right\} .
$$

The argument of Lemma 4.1 can be used to show that

$$
h \longrightarrow \int_{0}^{t \wedge \tau_{n}} \frac{G_{i}\left(h ; t, s, X_{s}\right)}{(t-s)^{\alpha / 2 q}} d W_{s}^{i}
$$

possesses an a.s. continuous modification on $\{|h| \leq N\}$. Since this is true for any $n$ and $N$, and $\cup_{n}\left\{\tau_{n} \geq t\right\}=\Omega$ a.s., the result follows.

(b) Existence: we want to show existence on an arbitrary interval $[0, T]$ ( $T$ will be fixed below).

Let $\left\{H^{n} ; n \in \mathbb{N}\right\}$ denote a progressively measurable localizing sequence for $H$ in $\left(L^{1,2}\right)^{p}$ on $[0, T]$. Since, from $\left(H .3^{\prime}\right), \sup _{t \leq T}\left|H_{t}\right|$ is a.s. finite, we can and do assume, without loss of generality, that

$$
\left|H_{t}^{n}(\omega)\right| \leq n, \quad \forall(t, \omega) \in[0, T] \times \Omega
$$

Note that $H_{t}(\omega)=H_{t}^{n}(\omega)$ a.s. on $\Omega_{n}^{T}$, for all $t \in[0, T]$, where $\Omega_{n}^{T} \uparrow \Omega$ a.s. as $n \rightarrow \infty$. We, moreover, define $X_{0}^{n}=X_{0} 1_{\left\{\left|X_{0}\right| \leq n\right\}}, S_{n}=\inf \left\{t ; \sup _{s<t}\left|D_{s} H_{t}^{n}\right| \vee V_{t}^{n} \geq n\right\}$. We consider the equation

$$
X_{t}^{n}=X_{0}^{n}+\int_{0}^{t} \tilde{F}^{n}\left(t, s, X_{s}^{n}\right) d s+\left.b \sum_{i=1}^{k} \int_{0}^{t} \frac{G_{i}^{n}\left(h ; t, s, X_{s}^{n}\right)}{(t-s)^{\alpha / 2 q}} d W_{s}^{i}\right|_{h=H_{t}^{n}}
$$

where

$$
\begin{gathered}
\tilde{F}^{n}(t, s, x)=1_{\left[0, S_{n}\right]}(s)\left[a \frac{F(t, s, x)}{(t-s)^{\alpha / q}}-b \sum_{i=1}^{k} \frac{G_{i}^{\prime}\left(H_{t}^{n} ; t, s, x\right) D_{s}^{i} H_{t}^{n}}{(t-s)^{\alpha / 2 q}}\right], \\
G_{i}^{n}(h ; t, s, x)=1_{\left[0, S_{n}\right]}(s) G_{i}(h ; t, s, x) .
\end{gathered}
$$

It is easy to see that theorem (4.15) applies to (5.6).

Define

$$
\bar{S}_{n}(\omega)= \begin{cases}S_{n}(\omega) \wedge \inf \left\{t \leq T ; \int_{0}^{t}\left|H_{s}(\omega)-H_{s}^{n}(\omega)\right| d s>0\right\} & \text { if }\left|X_{0}(\omega)\right|<n, \\ 0 & \text { otherwise. }\end{cases}
$$


$\bar{S}_{n}$ is a stopping time, and it follows from the uniqueness part of Theorem 4.4 that if $m>n$,

$$
X_{t}^{m}=X_{t}^{n} \quad \text { on }\left[0, \bar{S}_{n}\right] \text { a.s. }
$$

Since, moreover, $\left\{\bar{S}_{n}=T\right\} \uparrow \Omega$ a.s., we can define the process $\left\{X_{t}\right\}$ on $[0, T]$ by $X_{t}=X_{t}^{n}$ on $\left[0, \bar{S}_{n}\right]$, for all $n \in \mathbb{N}$.

Clearly, $X \in L^{q}(0, T)$ a.s. and solves $(3.1)$ on $[0, T]$. Since $T$ is arbitrary, the existence is proved.

(c) Uniqueness: it suffices to prove uniqueness on an arbitrary interval $[0, T]$. Let $\left\{\bar{X}_{t}, t \in[0, T]\right\}$ be a progressively measurable process such that $\bar{X} \in L^{q}(0, T)$ a.s. and $\bar{X}$ solves (3.1). It suffices to show that $\bar{X}$ coincides with the solution we have just constructed.

Let

$$
\begin{aligned}
\tilde{S}_{n}(\omega) & =\bar{S}(\omega) \wedge \inf \left\{t \leq T ; \int_{0}^{t} \frac{\left|\bar{X}_{s}(\omega)\right|^{q}}{(t-s)^{\alpha}} d s>n\right\}, \\
\tilde{X}_{t}^{n} & =\bar{X}_{t \wedge \tilde{S}_{n}} ;
\end{aligned}
$$

$\tilde{S}^{n} \in L^{q}(\Omega \times[0, T])$ and it solves (5.6) with $S_{n}$ replaced by $\tilde{S}_{n}$.

Then

$$
\tilde{X}_{t}^{n}(\omega)=X_{t}(\omega) \ell \times P \quad \text { a.e. on }\left[0, \tilde{S}_{n}\right]
$$

where $\ell$ is the Lebesgue measure on the real line.

The result follows from the fact that $\left\{\tilde{S}_{n} \geq T\right\} \uparrow \Omega$ a.s.

Note that the above solution satisfies, in fact, $X \in \bigcap_{q>1} \bigcap_{t>0} L^{q}(0, t)$ a.s.

\section{Continuity of the solution}

We want to give additional conditions under which the solution of (3.1) is an a.s. continuous process.

(H6) For all $(s, x) \in \mathbb{R}_{+} \times \mathbb{R}^{d}, t \rightarrow F(t, s, x) /(t-s)^{\alpha / q}$ is a.s. continuous on $(s,+\infty)$.

(H7) $\left\{H_{t} ; t \geq 0\right\}$ is a.s. continuous.

(H8) For all $i \in\{1, \ldots, k\}, s \in \mathbb{R}_{+}, t \rightarrow D_{s}^{i} H_{t}$ is a.s. continuous on $(s,+\infty)$.

(H9) For all $(s, x) \in \mathbb{R}_{+} \times \mathbb{R}^{d}, i \in\{1, \ldots, k\},(t, h) \rightarrow G_{i}^{\prime}(h ; t, s, x) /(t-s)^{\alpha / 2 q}$ is a.s. continuous on $(s,+\infty) \times \mathbb{R}^{P}$.

We also suppose that there exist $\delta>0, \ell>0$ such that for all $N>0,|h| \leq N, 0 \leq s<$ $t \wedge r, x \in \mathbb{R}^{d},|t-r| \leq 1$.

(H10) There exists an increasing process $\left\{V_{t}^{N} ; t \geq 0\right\}$ such that

$$
\left|G_{i}(h ; t, s, x)-G_{i}(h ; r, s, x)\right| \leq V_{t}^{N}|t-r|^{\delta}\left(1+|x|^{\ell}\right) .
$$

Theorem 6.1. Under conditions $\left(H 1^{\prime}\right),\left(H 2^{\prime}\right),\left(H 3^{\prime}\right),\left(H 4^{\prime}\right),\left(H 5^{\prime}\right)$ and $(H 6),(H 7),(H 8)$, (H9), (H10), the unique solution of (3.1) (which is progressively measurable and belongs a.s. to $\left.\bigcap_{q>1} \bigcap_{t>0} L^{q}(0, t)\right)$ has a.s. continuous modification. 
Proof. We need to show only that whenever $X \in \bigcap_{q>1} \bigcap_{t>0} L^{q}(0, t)$ a.s., $\left\{J_{t}(X) ; t>0\right\}$ has an a.s. continuous modification.

(a) We first show that $t \rightarrow \int_{0}^{t} \tilde{F}\left(t, s, X_{s}\right) d s$ is a.s. continuous.

Note that (H.6), (H.7), (H.8), and (H.9) imply that for all $(s, x) \in \mathbb{R}_{+} \times \mathbb{R}^{d}, t \rightarrow \tilde{F}(t, s, x)$ is a.s. continuous on $(s,+\infty)$.

Moreover, from $\left(\mathrm{H} .2^{\prime}\right),\left(\mathrm{H} .3^{\prime}\right),\left(\mathrm{H} .4^{\prime}\right)$, and the fact that $X \in \bigcap_{q>1} \bigcap_{t>0} L^{q}(0, t)$ a.s. for any $T>0$, there exists a process $\left\{Z_{s}^{T} ; s \in[0, T]\right\}$ such that

$$
\begin{aligned}
\left|F\left(t, s, X_{s}\right)\right| \leq & Z_{s}^{T}, \quad\left|G_{i}^{\prime}\left(H_{t} ; t, s, X_{s}\right) D_{s}^{i} H_{t}\right| \leq Z_{s}^{T}, \quad 0 \leq s<t \leq T \quad \text { a.s. }, \\
& \int_{0}^{t} Z_{s}^{T}\left\{\frac{1}{(t-s)^{\alpha / 2 q}}+\frac{1}{(t-s)^{\alpha / q}}\right\} d s<\infty \quad \text { a.s. }
\end{aligned}
$$

Let first $\left\{t_{n} ; n \in \mathbb{N}\right\}$ be a sequence such that $t_{n}<t$ for any $n$ and $t_{n} \rightarrow t$ as $n \rightarrow \infty$; then

$$
\begin{aligned}
\int_{0}^{t} \tilde{F}\left(t, s, X_{s}\right) d s- & \int_{0}^{t_{n}} \tilde{F}\left(t_{n}, s, X_{s}\right) d s=\int_{t_{n}}^{t} \tilde{F}\left(t, s, X_{s}\right) d s+\int_{0}^{t_{n}}\left[\tilde{F}\left(t, s, X_{s}\right)-\tilde{F}\left(t_{n}, s, X_{s}\right)\right] d s, \\
\mid & \int_{t_{n}}^{t} \tilde{F}\left(t, s, X_{s}\right) d s \mid \leq \int_{t_{n}}^{t} Z_{s}^{T}\left\{\frac{1}{(t-s)^{\alpha / 2 q}}+\frac{1}{(t-s)^{\alpha / q}}\right\} d s,
\end{aligned}
$$

and the latter tends a.s. to 0 as $n \rightarrow \infty$.

$$
\left|\int_{0}^{t_{n}}\left[\tilde{F}\left(t, s, X_{s}\right)-\tilde{F}\left(t_{n}, s, X_{s}\right)\right] d s\right| \leq \int_{0}^{t}\left|\tilde{F}\left(t, s, X_{s}\right)-\tilde{F}\left(t_{n}, s, X_{s}\right)\right| d s
$$

which tends to 0 as $n \rightarrow \infty$. A similar argument gives the same result when $t_{n}>t, t_{n} \rightarrow t$.

(b) We next show that $t \rightarrow I_{t}\left(X, H_{t}\right)$ possesses an a.s. continuous modification. This follows from (H.7) and

$$
(t, h) \longrightarrow I_{t}(X, h)
$$

has an a.s. continuous modification.

By localization, it suffices to prove (6.5) under assumptions (H.2), (H.3), (H.4), (H.5) and (H.6), (H.7), (H.8), (H.9), (H.10), with $V_{t}^{N}(\omega)$ in (H.10) replaced by a constant $K$, and in case $X_{0} \in \bigcap_{q>1} L^{q}\left(\Omega ; \mathbb{R}^{d}\right)$.

It then suffices to show that under the above hypotheses, there exists $C, q>0$ such that for any $h, k \in \mathbb{R}^{P}$, the numbers $t, r$ will be positive. Suppose, to fix the ideas, that $0 \leq r<t$; then

$$
\begin{aligned}
I_{t}(X, h)-I_{r}(X, k)= & \int_{r}^{t} \frac{G_{i}\left(h ; t, s, X_{s}\right)}{(t-s)^{\alpha / 2 q}} d W_{s}^{i}+\int_{0}^{r}\left[\frac{G_{i}\left(h ; t, s, X_{s}\right)}{(t-s)^{\alpha / 2 q}}-\frac{G_{i}\left(h ; r, s, X_{s}\right)}{(r-s)^{\alpha / 2 q}}\right] d W_{s}^{i} \\
& +\int_{0}^{r}\left[\frac{G_{i}\left(h ; t, s, X_{s}\right)}{(r-s)^{\alpha / 2 q}}-\frac{G_{i}\left(k ; r, s, X_{s}\right)}{(r-s)^{\alpha / 2 q}}\right] d W_{s}^{i} .
\end{aligned}
$$


It follows from the Burkholder-Gundy inequality that

$$
\begin{aligned}
E\left(\left|\int_{r}^{t} \frac{G_{i}\left(h ; t, s, X_{s}\right)}{(t-s)^{\alpha / 2 q}} d W_{s}^{i}\right|^{q}\right) & \leq c_{q} \sum_{i=1}^{k} E\left[\left(\int_{r}^{t} \frac{\left|G_{i}\left(h ; t, s, X_{s}\right)\right|^{2}}{(t-s)^{\alpha / q}} d s\right)^{q / 2}\right] \\
& \leq c_{q}(t-r)^{(q-2) / 2} \sum_{i=1}^{k} E \int_{r}^{t} \frac{\left|G_{i}\left(h ; t, s, X_{s}\right)\right|^{q}}{(t-s)^{\alpha / 2}} d s \\
& \leq c_{q}(t-r)^{(q-2) / 2} \int_{r}^{t} \frac{E\left(1+\left|X_{s}\right|^{q}\right)}{(t-s)^{\alpha / 2}} d s \\
& \leq C_{q}(t-r)^{(q-2) / 2}(t-r)^{1-\alpha / 2} .
\end{aligned}
$$

From (H4), for $G_{i}$, we deduce, as in Lemma 4.1, that

$$
\begin{aligned}
& E\left(\left|\int_{0}^{r}\left[\frac{G_{i}\left(h ; r, s, X_{s}\right)-G_{i}\left(k ; r, s, X_{s}\right)}{(r-s)^{\alpha / 2 q}}\right] d W_{s}^{i}\right|^{q}\right) \\
& \quad \leq c_{q}(h-k)^{q} \int_{0}^{r} \frac{E\left(1+\left|X_{s}\right|^{q}\right)}{(r-s)^{\alpha / 2}} d s \leq C_{q}(h-k)^{q} r^{1-\alpha / 2} .
\end{aligned}
$$

From (H.10) and the fact that

$$
\begin{aligned}
& X \in \bigcap_{q>1} \bigcap_{t>0} L^{q}(\Omega \times(0, t)), \\
& E\left(\left|\int_{0}^{r}\left[\frac{G_{i}\left(h ; t, s, X_{s}\right)}{(t-s)^{\alpha / 2 q}}-\frac{G_{i}\left(h ; r, s, X_{s}\right)}{(r-s)^{\alpha / 2 q}}\right] d W_{s}^{i}\right|^{q}\right) \\
& =E\left(\mid \int_{0}^{r}\left(\frac{1}{(r-s)^{\alpha / 2 q}}\left[G_{i}\left(h ; t, s, X_{s}\right)-G_{i}\left(h ; r, s, X_{s}\right)\right]\right.\right. \\
& \left.\left.+G_{i}\left(h ; t, s, X_{s}\right)\left[\frac{1}{(t-s)^{\alpha / 2 q}}-\frac{1}{(r-s)^{\alpha / 2 q}}\right]\right)\left.d W_{s}^{i}\right|^{q}\right) \\
& \leq E\left(c _ { q } \int _ { 0 } ^ { r } \left(\frac{\left|G_{i}\left(h ; t, s, X_{s}\right)-G_{i}\left(h ; r, s, X_{s}\right)\right|^{q}}{(r-s)^{\alpha}}\right.\right. \\
& \left.\left.+\left|G_{i}\left(h ; t, s, X_{s}\right)\right|^{q}\left((r-s)^{-\alpha / 2 q}-(t-s)^{-\alpha / 2 q}\right)^{q}\right) d s\right) \\
& \leq c_{q} K^{q}\left(\int_{0}^{r}\left(\frac{|t-r|^{\delta q}}{(r-s)^{\alpha}} E\left(1+\left|X_{s}\right|^{q \ell}\right)\right) d s\right. \\
& \left.+\int_{0}^{r}\left(E\left(1+\left|X_{s}\right|^{q}\right)\left((r-s)^{-\alpha / 2}-(t-s)^{-\alpha / 2}\right)\right) d s\right) \\
& \leq \tilde{c}_{q}\left[|t-r|^{\delta q} \int_{0}^{r}(r-s)^{-\alpha / 2} d s+\int_{0}^{r}\left((r-s)^{-\alpha / 2}-(t-s)^{-\alpha / 2}\right) d s\right] \\
& \leq C_{q}\left(|t-r|^{\delta q} r^{1-\alpha / 2}+\left(r^{1-\alpha / 2}+(t-r)^{1-\alpha / 2}-t^{1-\alpha / 2}\right)\right)
\end{aligned}
$$


which from the above estimate yields

$$
\begin{aligned}
& E\left(\left|I_{t}(X, h)-I_{r}(X, k)\right|^{q}\right) \\
& \quad \leq C_{q}\left[|t-r|^{1-\alpha / 2}+|t-r|^{(q-\alpha) / 2}\right. \\
& \left.\quad+r^{1-\alpha / 2}\left(|t-r|^{\delta q}+|h-k|^{q}\right)+\left(r^{1-\alpha / 2}-t^{1-\alpha / 2}\right)\right]
\end{aligned}
$$

this completes the proof.

\section{Acknowledgment}

We are very grateful to the referees for valuable comments.

\section{References}

[1] C. Bender and R. J. Elliott, Binary market models and discrete wick products, preprint, 2002.

[2] M. A. Berger and V. J. Mizel, Volterra equations with Itô integrals. I, J. Integral Equations 2 (1980), no. 3, 187-245.

[3] - Volterra equations with Itô integrals. II, J. Integral Equations 2 (1980), no. 4, 319-337.

[4] An extension of the stochastic integral, Ann. Probab. 10 (1982), no. 2, 435-450.

[5] M. M. El-Borai, Some probability densities and fundamental solutions of fractional evolution equations, Chaos Solitons Fractals 14 (2002), no. 3, 433-440.

[6] A. M. Kolodh, On the existence solutions of stochastic Volterra equations, Theory of Random Processes 11 (1983), 51-57 (Russian).

[7] B. B. Mandelbrot and J. W. Van Ness, Fractional Brownian motions, fractional noises and applications, SIAM Rev. 10 (1968), 422-437.

[8] P.-A. Meyer, Flot d'une équation différentielle stochastique (d'après Malliavin, Bismut, Kunita) [Flow of a stochastic differential equation (following Malliavin, Bismut, Kunita)], Seminar on Probability, XV (University of Strasbourg, Strasbourg, 1979/1980), Lecture Notes in Math., vol. 850, Springer-Verlag, Berlin, 1981, pp. 103-117 (French).

[9] D. Nualart, Noncausal stochastic integrals and calculus, Stochastic Analysis and Related Topics (Silivri, 1986), Lecture Notes in Math., vol. 1316, Springer-Verlag, Berlin, 1988, pp. 80-129.

[10] D. Nualart and É. Pardoux, Stochastic calculus with anticipating integrands, Probab. Theory Related Fields 78 (1988), no. 4, 535-581.

[11] D. Nualart and M. Zakai, Generalized stochastic integrals and the Malliavin calculus, Probab. Theory Related Fields 73 (1986), no. 2, 255-280.

[12] D. Ocone and É. Pardoux, A generalized Itô-Ventzell formula. Application to a class of anticipating stochastic differential equations, Ann. Inst. H. Poincaré Probab. Statist. 25 (1989), no. 1, $39-71$.

[13] Linear stochastic differential equations with boundary conditions, Probab. Theory Related Fields 82 (1989), no. 4, 489-526.

[14] S. Ogawa, Sur la question d'existence de solutions d'une équation différentielle stochastique du type noncausal [On the existence of solutions of a stochastic differential equation of noncausal type], J. Math. Kyoto Univ. 24 (1984), no. 4, 699-704 (French).

[15] É. Pardoux and P. Protter, A two-sided stochastic integral and its calculus, Probab. Theory Related Fields 76 (1987), no. 1, 15-49.

[16] Stochastic Volterra equations with anticipating coefficients, Ann. Probab. 18 (1990), no. 4, 1635-1655.

[17] P. Protter, Volterra equations driven by semimartingales, Ann. Probab. 13 (1985), no. 2, 519530. 


\section{Volterra equations with fractional stochastic integrals}

[18] A. N. V. Rao and C. P. Tsokos, On the existence, uniqueness, and stability behavior of a random solution to a nonlinear perturbed stochastic integro-differential equation, Information and Control 27 (1975), 61-74.

[19] Y. Shiota, A linear stochastic integral equation containing the extended Itô integral, Math. Rep. Toyama Univ. 9 (1986), 43-65.

[20] A. V. Skorohod, On a generalization of the stochastic integral, Teor. Verojatnost. i Primenen. 20 (1975), no. 2, 223-238 (Russian).

[21] A.-S. Sznitman, Martingales dépendant d'un paramètre: une formule d'Itô [Martingales depending on a parameter: an Ito formula], Z. Wahrsch. Verw. Gebiete 60 (1982), no. 1, 41-70 (French).

[22] W. Wyss, The fractional diffusion equation, J. Math. Phys. 27 (1986), no. 11, 2782-2785.

Mahmoud M. El-Borai: Faculty of Science, Alexandria University, Alexandria, Egypt E-mail address: m_m_elborai@yahoo.com

Khairia El-Said El-Nadi: Faculty of Science, Alexandria University, Alexandria, Egypt E-mail address: khairia_el_said@hotmail.com

Osama L. Mostafa: Faculty of Science, Alexandria University, Alexandria, Egypt E-mail address: olmoustafa@yahoo.com

Hamdy M. Ahmed: Faculty of Science, Alexandria University, Alexandria, Egypt E-mail address: hamdy_17eg@yahoo.com 


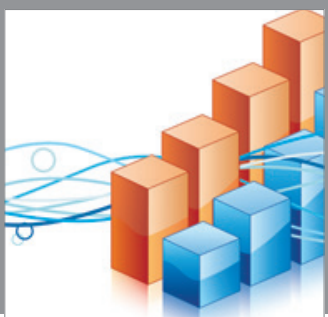

Advances in

Operations Research

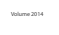

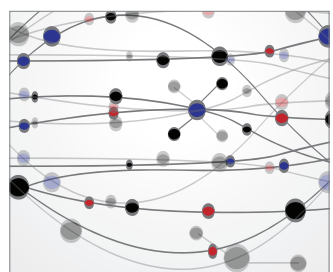

\section{The Scientific} World Journal
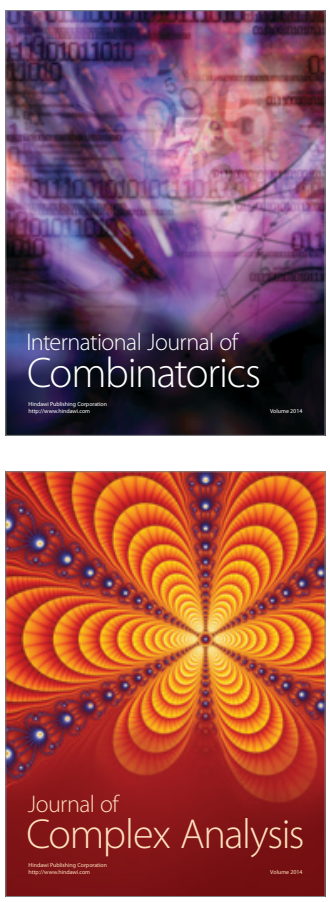

International Journal of

Mathematics and

Mathematical

Sciences
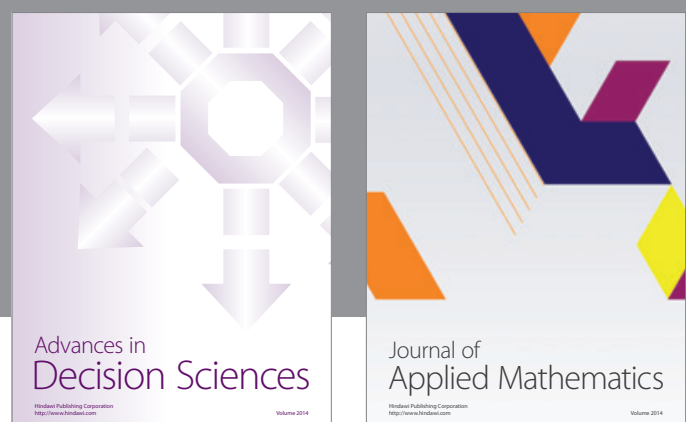

Journal of

Applied Mathematics
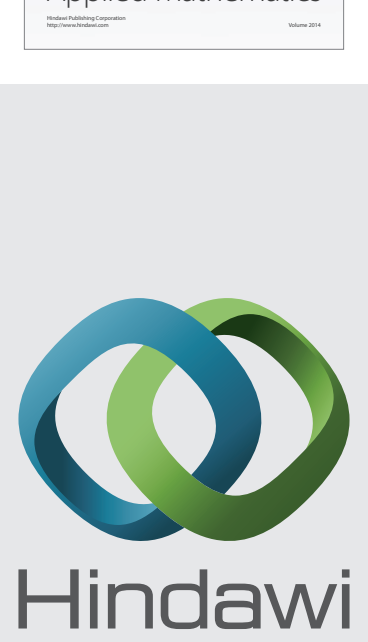

Submit your manuscripts at http://www.hindawi.com
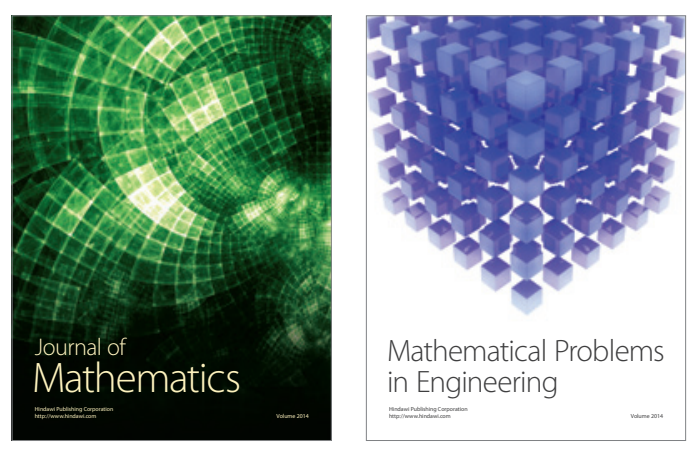

Mathematical Problems in Engineering
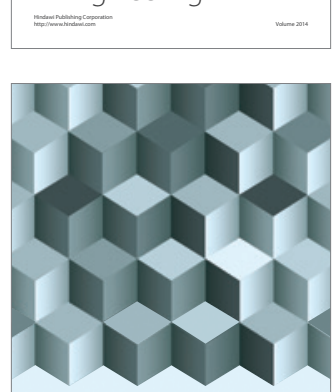

Journal of

Function Spaces
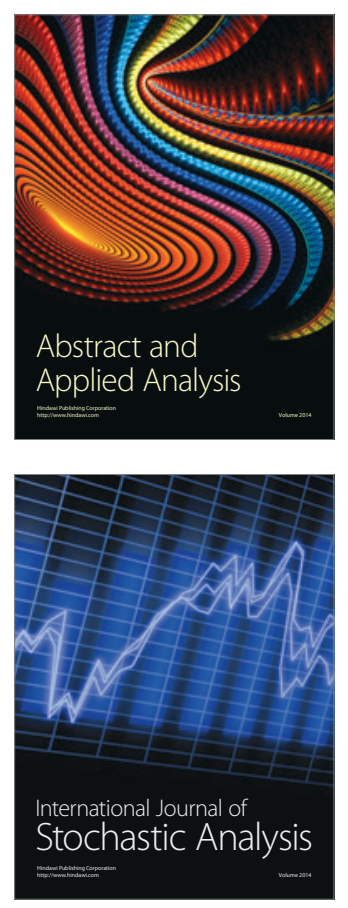

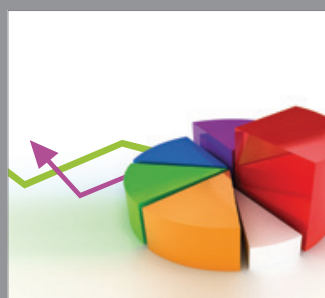

ournal of

Probability and Statistics

Promensencen
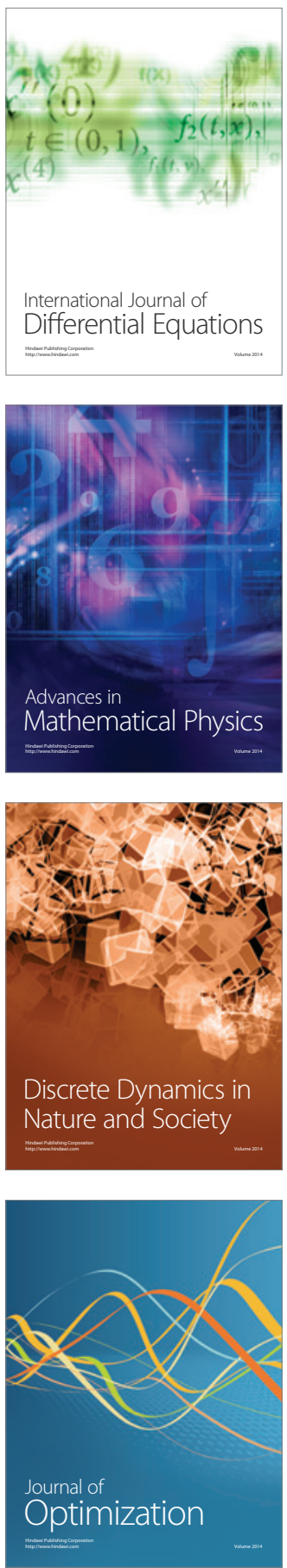